\title{
Family Nurse Practitioner
}

National Cancer Institute

\section{Source}

National Cancer Institute. Family Nurse Practitioner. NCI Thesaurus. Code C71290.

A reg istered nurse with specialized and advanced education in treating all age groups.

The Family Nurse Practitioner can treat both pediatric and adult members of the family who suffer from acute or chronic illnesses, as well as address psychosocial issues that may be affecting the family. 